\title{
In Search of a Dose: The Functional and Molecular Effects of Exercise on Post-stroke Rehabilitation in Rats
}

\author{
Fengwu Li ${ }^{1}$, Xiaokun Geng ${ }^{1,2,3 *}$, Christian Huber ${ }^{3}$, Christopher Stone ${ }^{3}$ \\ and Yuchuan Ding $3,4 *$
}

${ }^{1}$ China-America Institute of Neuroscience, Luhe Hospital, Capital Medical University, Beijing, China, ${ }^{2}$ Department of Neurology, Beijing Luhe Hospital, Capital Medical University, Beijing, China, ${ }^{3}$ Department of Neurosurgery, Wayne State University School of Medicine, Detroit, MI, United States, ${ }^{4}$ Department of Research and Development Center, John D. Dingell VA Medical Center, Detroit, MI, United States

OPEN ACCESS

Edited by:

Claudio Rivera,

Aix-Marseille Université, France

Reviewed by:

Jérôme Laurin,

Aix-Marseille Université, France

Anne Sophie Tessier,

INSERM U1093 Cognition, Action et

Plasticité Sensomotrice, France

*Correspondence:

Xiaokun Geng

xgeng@ccmu.edu.cn

Yuchuan Ding

yding@med.wayne.edu

Specialty section:

This article was submitted to Cellular Neuropathology, a section of the journal Frontiers in Cellular Neuroscience

Received: 01 November 2019 Accepted: 28 May 2020

Published: 25 June 2020

Citation:

Li F, Geng X, Huber C, Stone $C$ and Ding Y (2020) In Search of a Dose: The Functional and Molecular Effects of Exercise on Post-stroke Rehabilitation in Rats. Front. Cell. Neurosci. 14:186. doi: 10.3389/fncel.2020.00186
Although physical exercise has been demonstrated to augment recovery of the post-stroke brain, the question of what level of exercise intensity optimizes neurological outcomes of post-stroke rehabilitation remains unsettled. In this study, we aim to clarify the mechanisms underlying the intensity-dependent effect of exercise on neurologic function, and thereby to help direct the clinical application of exercisebased neurorehabilitation. To do this, we used a well-established rat model of ischemic stroke consisting of cerebral ischemia induction through middle cerebral artery occlusion (MCAO). Ischemic rats were subsequently assigned either to a control group entailing post-stroke rest or to one of two exercise groups distinguished by the intensity of their accompanying treadmill regimens. After $24 \mathrm{~h}$ of reperfusion, exercise was initiated. Infarct volume, apoptotic cell death, and neurological defects were quantified in all groups at 3 days, and motor and cognitive functions were tracked up to day-28. Additionally, Western blotting was used to assess the influence of our interventions on several proteins related to synaptogenesis and neuroplasticity (growth-associated protein 43, a microtubule-associated protein, postsynaptic density-95, synapsin I, hypoxia-inducible factor- $1 \alpha$, brain-derived neurotrophic factor, nerve growth factor, tyrosine kinase $B$, and cAMP response element-binding protein). Our results were in equal parts encouraging and surprising. Both mild and intense exercise significantly decreased infarct volume, cell death, and neurological deficits. Motor and cognitive function, as determined using an array of tests such as beam balance, forelimb placing, and the Morris water maze, were also significantly improved by both exercise protocols. Interestingly, while an obvious enhancement of neuroplasticity proteins was shown in both exercise groups, mild exercise rats demonstrated a stronger effect on the expressions of Tau $(p<0.01$ ), brain-derived neurotrophic factor $(p<0.01)$, and tyrosine kinase B $(p<0.05)$. These findings contribute to the growing body of literature regarding the positive effects of both mild and intense long-term treadmill exercise on brain injury, functional outcome, and 


\begin{abstract}
neuroplasticity. Additionally, the results may provide a base for our future study regarding the regulation of $\mathrm{HIF}-1 \alpha$ on the BDNF/TrkB/CREB pathway in the biochemical processes underlying post-stroke synaptic plasticity.
\end{abstract}

Keywords: ischemia/reperfusion, functional outcome, synaptogenesis, BDNF, TrkB, CREB, HIF-1 $\alpha$

\section{INTRODUCTION}

Exercise therapy has long been considered a promising strategy to ameliorate physical disability after stroke (Saposnik et al., 2016). However, neurological outcomes of post-stroke rehabilitation appear to differ according to the intensity of the exercise regimen that is used (Bell et al., 2015; Xing et al., 2018). Some previous studies have demonstrated that higher intensity exercise may yield better functional recovery and neuroplasticity (Linder et al., 2019; Luo et al., 2019; Andrews et al., 2020), while other studies have suggested that mild exercise results in superior neuroprotection and synaptic plasticity after stroke (Lee et al., 2009; Shih et al., 2013). These conflicting results underscore the principle that exercise intensity is an important determinant of post-stroke neurological outcomes. Therefore, clarifying the mechanisms that underlie an intensity-dependent effect of exercise on neurologic function may help direct the clinical application of exercise-based neurorehabilitation. Currently, these mechanisms have not been fully explored, and are consequently incompletely understood.

Recently, brain-derived neurotrophic factor (BDNF) has become the subject of increasing attention as a possible mediator of the neurological benefits of exercise. BDNF is an abundant growth factor that is involved in activity-induced neuroplasticity and is upregulated in the animal brain by exercise. The regulation of neuroplasticity depends on a complex set of interactions between a variety of neural proteins, including postsynaptic density 95 (PSD-95; Wang et al., 2019), synapsin I (SYN; Pan et al., 2017), growth-associated protein 43 (GAP-43), and microtubule-associated protein (also known as Tau; Biundo et al., 2018; Mercerón-Martínez et al., 2018; Pu et al., 2019). Changes in these neuroplastic factors are related to exerciseinduced activation of BDNF (Kim and Leem, 2016; Belviranli and Okudan, 2019). Previous research has suggested a pivotal regulatory role for BDNF and its receptor, BDNF-tyrosine kinase $\mathrm{B}(\operatorname{TrkB})$, regarding neuroplasticity after physical exercise (Lee et al., 2018), mediated through the expression of the transcription factor cyclic AMP response element-binding protein (CREB; $\mathrm{Hu}$ et al., 2004). Moreover, activation of the BDNF/TrkB/CREB signaling pathway has also been shown to promote functional recovery after stroke (Liu H. et al., 2018). Taken together, these lines of evidence suggest that post-stroke exercise regimens such as the one used in this study may induce neuroplasticity and influence rehabilitative outcomes through the changes they provoke in the BDNF pathway.

Another factor that may be involved in determining the outcomes of post-stroke exercise regimens is hypoxia-inducible factor- $1 \alpha$ (HIF-1 $\alpha)$. Upregulation of HIF- $1 \alpha$ by exercise has been reported to play a role in reducing infarct volumes following ischemia/reperfusion injury (Li C. et al., 2017), and in post-stroke neuroplasticity (Wu et al., 2018). Previous studies demonstrated that HIF-1 $\alpha$ also induced the expression of BDNF (Shi et al., 2009; Nakamura et al., 2011; Helan et al., 2014), and thereby promoted neuroplasticity, reduced neuronal death, and improved neurological function in a rat model of ischemic stroke (Chen et al., 2017). HIF-1 $\alpha$ has further been shown to stimulate the expression of the TrkB receptor (Martens et al., 2007), and the CREB receptor in various cancer cells (Yu et al., 2020). However, despite this considerable circumstantial evidence, previous studies have not yet explored the effect of HIF- $1 \alpha$ on BDNF/TrkB/CREB pathway in improving synaptic plasticity following ischemia/reperfusion injury. Although the present study did not determine this relation, as the first step, we intended to assess the expression of HIF- $1 \alpha$ and $\mathrm{BDNF} / \mathrm{TrkB} / \mathrm{CREB}$ proteins following ischemia/reperfusion injury. These results might suggest a potential association of these molecules and provide a base for our future study regarding the regulation of HIF- $1 \alpha$ on the BDNF/TrkB/CREB pathway.

\section{MATERIALS AND METHODS}

\section{Animals}

A total of 150 adult male Sprague-Dawley rats (280-300 g, Vital River Laboratory Animal Technology Company Limited, Beijing, China) were used in this study. The protocol by which they were studied was approved by the Animal Care and Use Committee of Capital Medical University, and the study was conducted following the NIH Guide for the Care and Use of Laboratory Animals. Animals were randomly divided into three groups: middle cerebral artery occlusion (MCAO) without exercise (50), MCAO plus intense treadmill exercise (50), and MCAO plus mild treadmill exercise (50). Both exercise protocols were initiated after $24 \mathrm{~h}$ reperfusion, and animals in each group were sacrificed at days 3, 14, and 28 after reperfusion for further biochemical analysis.

\section{Focal Cerebral Ischemia}

The animals were subjected to transient right MCAO according to the method we described previously (Li F. et al., 2019). Briefly, rats were anesthetized in a chamber using 3\% isoflurane and a mixture of $70 \%$ nitrous oxide and $30 \%$ oxygen. Then rats were then transferred to a surgical table, where anesthesia was maintained with a facemask that delivered $1 \%$ isoflurane from a calibrated precision vaporizer, and poly-L-lysine-coated nylon (4.0) sutures were used to generate infarcts with minimal inter-animal variability. During the unilateral, 2-h MCAO procedure, cerebral blood flow $(\mathrm{CBF})$, blood $\mathrm{pCO}_{2}$ and $\mathrm{pO}_{2}$, mean arterial pressure (MAP), and rectal temperature were monitored continuously. Rectal temperatures were maintained between $36.5^{\circ} \mathrm{C}$ and $37.5^{\circ} \mathrm{C}$ using a heating pad and a heating 
lamp. Ipsilesional ischemic cerebral hemispheres were used for molecular analysis.

\section{Treadmill Exercise}

Animals were randomly assigned either to the exercise groups or the non-exercise control group. Exercise animals were run on a four-lane treadmill (ZS-PT-II, ZS Dichuang Instruments, Inc., Beijing, China), either at a constant speed of $30 \mathrm{~m} / \mathrm{min}$ for $30 \mathrm{~min}$ each day (intense); or at $5 \mathrm{~m} / \mathrm{min}$ for the first $10 \mathrm{~min}, 9 \mathrm{~m} / \mathrm{min}$ for the second $10 \mathrm{~min}$, and $12 \mathrm{~m} / \mathrm{min}$ for the last $10 \mathrm{~min}$ on days 1 and 2, followed by $12 \mathrm{~m} / \mathrm{min}$ on the third and subsequent days (mild). The mild exercise was begun at a shorter intensity (days 1-2) and ultimately ended with the final mild speed at 3 days and thereafter, such that low intensity was maintained throughout. This gradual start could not be achieved for the intense exercise group, however, as we would, in this case, have been unable to accurately assess the effects of high intensity in the short-term (3 days) when rats were sacrificed. Both exercise and non-exercise animals were housed in groups of three in standard cages for equal time.

\section{Neurological Deficit}

The modified scoring systems proposed by Zea Longa (5-point) and Belayev et al. (1996) (12-point) were used to examine the severity of neurological deficits in rats before and after $24 \mathrm{~h}$ reperfusion (Li F. et al., 2019). After MCAO, rats with scores of 2 or below were considered to represent the unsuccessful establishment of the MCAO model and were consequently excluded (about 10\%); exclusion was then confirmed on autopsy by lack of a core, indicating a faulty surgery.

\section{Cerebral Infarct Volume}

At 3 days of ischemia and reperfusion in rats that underwent $\mathrm{MCAO}$, brains were resected and cut into 2-mm-thick slices, which were then treated with 2,3,5-triphenyltetrazolium chloride (TTC; Sigma-Aldrich, St. Louis, MO, USA) for staining (Li et al., 2017b), facilitating the use of an indirect method for calculating infarct volume to minimize error caused by edema.

\section{Apoptotic Cell Death}

For quantification of apoptosis-related DNA fragmentation, a commercial enzyme immunoassay was used to determine cytoplasmic histone-associated DNA fragments (Cell Death Detection ELISA; mlbio, Shanghai, China). The degree of apoptosis was quantified according to the amount of cytoplasmic histone-associated DNA fragments in the control and experimental groups at 3 days after physical exercise.

\section{Neurobehavioral Tests}

These tests included adhesive removal, beam balance, forelimb placing, grid walking, and Rota-rod performance (R03-1; Xin-Ruan Instruments, Inc., Shanghai, China), as assessed at days 1, 3, 7, 14, 21, and 28. The Morris water maze (ZS-II; ZS Dichuang Instruments, Inc., Beijing, China) was also employed, in our case at 24-28 days; this is following a previous report that showed no obvious suppressive effect on swimming at 24 days after the ischemic event (Ran et al., 2018). In the adhesive removal test, the tape was attached to the palmar surface of the forepaw, and the time taken for the first attempt to touch and to remove the tape was recorded. In the beam balance test, rats were placed on a narrow wooden beam $(122 \times 2.5 \times 42 \mathrm{~cm})$, and performance was scored from 0 to $6(0=$ no attempt to stay on the beam; 1 = attempted to stay on the beam but no movement; $2=$ attempted to cross the beam but failed; 3 = crossed the beam with contralateral hindlimb slips $>50 \%$ of the time; $5=$ crossed the beam with contralateral hindlimb slips $<50 \%$ of the time; $6=$ crossed the beam without slips; see Ran et al. (2018). In the forelimb placing test, rats were held gently with forelimbs close to the tabletop while the surface was lightly brushed using each side of their vibrissa. The ability of rats to place the preferred forelimb on the edge of the table in this context was recorded 10 times, and placing rates were calculated. In the grid walk test, rats were placed on a wire grid $(100 \times 25 \times 50 \mathrm{~cm})$ and allowed to walk from one end to the other; the total number of foot slips during this crossing was recorded. In the Rota-rod test, rats were placed on a rotating drum that accelerated from 4 to $40 \mathrm{rpm}$ within $300 \mathrm{~s}$; the time that the animals stayed on the rotating rod was then recorded. Finally, in the Morris water maze, rats were placed into a pool (diameter $=150 \mathrm{~cm}$ ) at one of the four locations, and allowed to swim for $90 \mathrm{~s}$ to find a hidden platform (diameter $=10 \mathrm{~cm}$ ); swim speed and the time taken to find the hidden platform were recorded using a camera positioned above the pool that transmitted data to an analysis system for calculation. Tests were conducted on non-consecutive days to mitigate possible confounding due to motor learning that might have occurred if these tests were performed in close succession.

\section{Neuron Isolation and Flow Cytometry Assay}

As described previously by our group (Chen et al., 2018), an adult brain dissociation kit (Miltenyi Biotec, Bergisch Gladbach, Germany) was used for neuron isolation. Briefly, rats were sacrificed at 3,14 , and 28 days after exercise and ipsilesional brains were finely cut, ground, and filtered through a $70-\mu \mathrm{m}$ cell strainer (Miltenyi Biotec, Bergisch Gladbach, Germany) to obtain a single-cell suspension. Cell pellets $\left(5 \times 10^{6}\right.$ cells $)$ were stained with primary antibodies against Tau or GAP-43 $\left(1 \mu \mathrm{g} / 1 \times 10^{6}\right.$ cells, rabbit anti-Tau, and rabbit anti-GAP43, Abcam, MA, USA) in darkness for $30 \mathrm{~min}$ at room temperature. Cells were washed three times with PBS and incubated with Alexa Fluor ${ }^{\circledR} 488$ fluorescein-conjugated secondary antibodies (Sigma, St. Louis, MO, USA) for $30 \mathrm{~min}$ at room temperature, and then washed again and analyzed on a FACS Calibur flow cytometer (Accuri C6, BD, San Jose, CA, USA) with Cell Quest software (BD, San Jose, CA, USA).

\section{Protein Expression}

At 3, 14, and 28 days after initiation of the exercise regimens, rats were sacrificed for Western blot analysis. Tissue samples from the ipsilesional ischemic cerebral hemispheres of all experimental groups were harvested, and total protein extraction was performed using cell lysis solutions (Thermo Fisher Scientific, Inc., Waltham, MA, USA). Protein concentration was then determined by the BCA method. Electrophoresis (10\% SDS-PAGE gel) was performed 

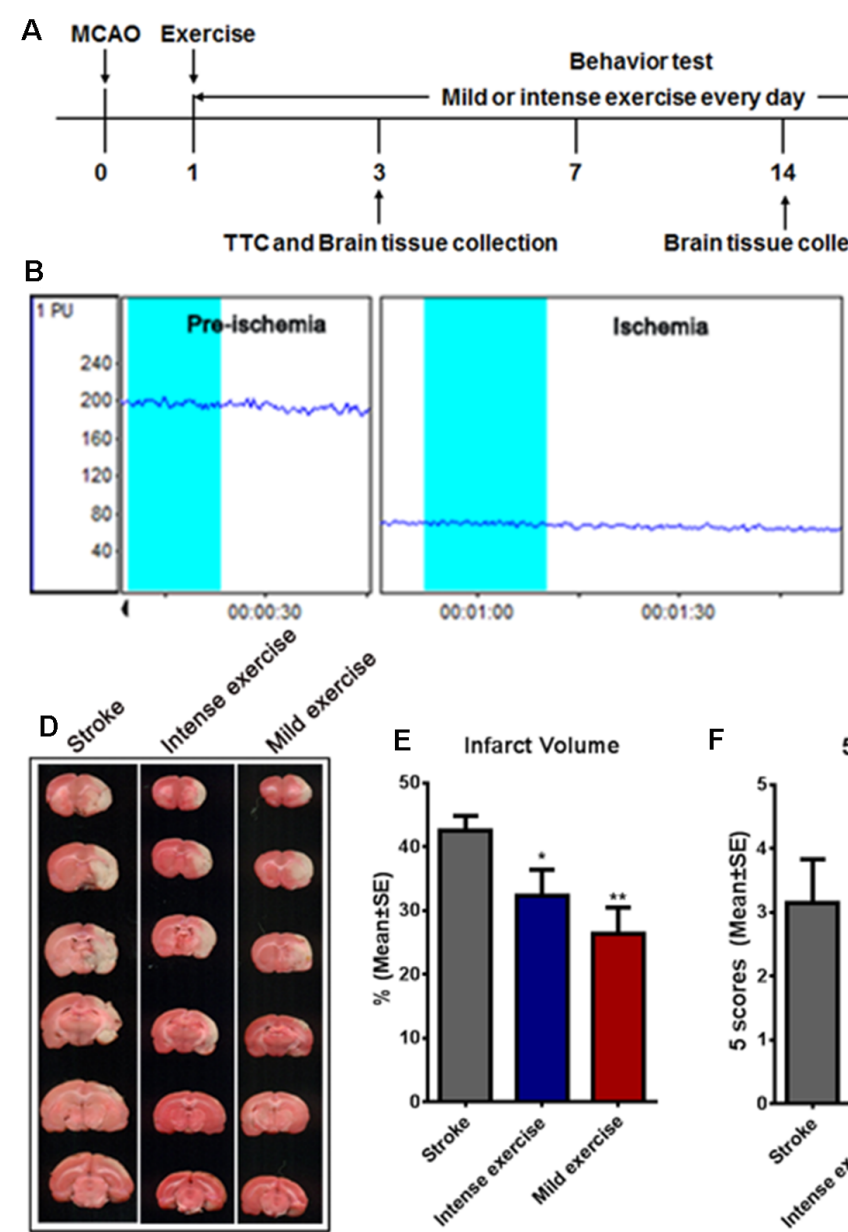

$\mathbf{F}$
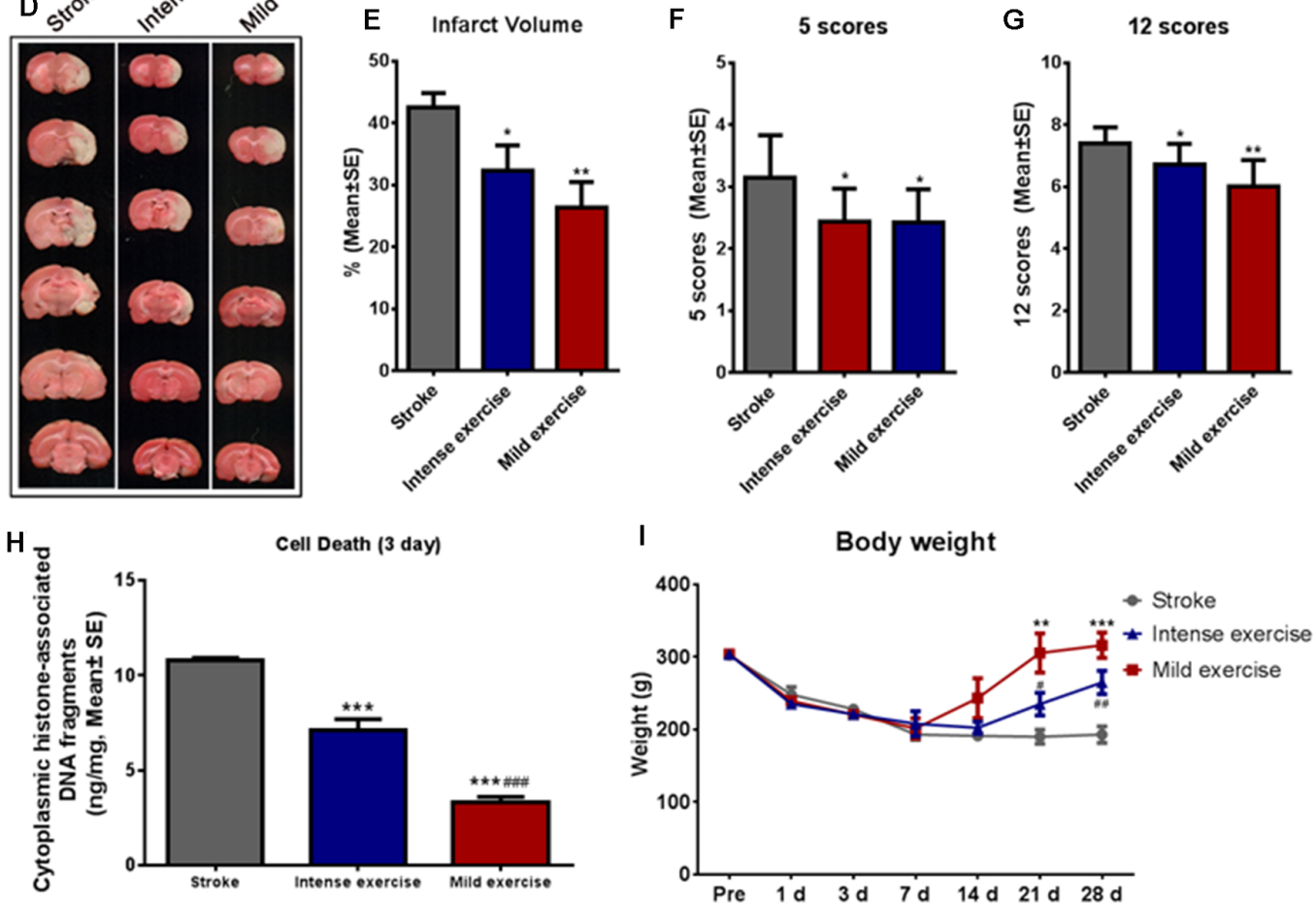

FIGURE 1 | Mild or intense exercise reduced brain infarct. (A) Illustration of the experimental timelines. Rats were subjected to $2 \mathrm{~h}$ middle cerebral artery occlusion (MCAO), followed by daily treadmill exercise 1 day after reperfusion for up to 28 days. (B,C) Representative images and quantification of cerebral blood flow (CBF) monitoring of the three study groups for 2 min before and after the onset of ischemia. There were no significant differences in CBF between groups. (D)

2,3,5-triphenyltetrazolium chloride (TTC) histology demonstrating exercise-induced infarct volume reduction in the penumbra region of the ischemic territory supplied by the middle cerebral artery. (E) Quantification of the infarct volume reduction exercise. Both mild $\left({ }^{\star \star} p<0.01\right)$ and intense $\left({ }^{\star} p<0.05\right)$ exercise significantly decreased infarct volumes, but the reduction was more pronounced with mild exercise. Neurological deficits were tracked after both types of exercise using both the 5- (F) and 12- (G) point systems. ANOVA analyses indicated that both mild $\left({ }^{* *} p<0.01\right)$ and intense exercise $\left({ }^{*} p<0.05\right)$ reduced neurological deficits. (H) Cell death reduction due to exercise quantified at 3 days. Both mild and intense exercise reduced apoptotic cell death significantly $\left({ }^{\star \star \star} p<0.001\right)$, but a more significant

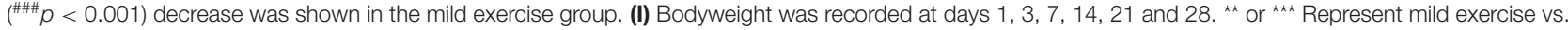
control; " or \#\# represent intense exercise vs. control.

with $30 \mu \mathrm{g}$ of protein per lane. Gel transfer to a PVDF membrane was performed under $200 \mathrm{~V}$ for $1 \mathrm{~h}$. Membranes were blocked with $5 \%$ skimmed milk, followed by incubation with primary antibodies (1:1,000 rabbit anti-BDNF, rabbit
anti-NGF, rabbit anti-PSD-95, rabbit anti-SYN, rabbit antiTau, and rabbit anti-GAP43, Abcam, MA, USA; 1:500 rabbit anti-HIF-1 $\alpha$, Santa Cruz Biotechnology, Inc., Santa Cruz, CA, USA) overnight at $4^{\circ} \mathrm{C}$. The next day, membranes were 
washed three times and further incubated with a goat anti-rabbit IgG-HRP secondary antibody (1:1,000, Santa Cruz) at room temperature for $1 \mathrm{~h}$. After washing, the ECL method was used to detect signals. Western blot images for each antibody were analyzed using an image analysis program (ImageJ 1.42, National Institutes of Health, Bethesda, MD, USA) to quantify protein expression according to relative image density.

\section{Statistical Analysis}

Statistical analyses were performed with SPSS Statistics for Windows, Version 17.0 (SPSS Inc., Chicago, IL, USA). Differences among groups were assessed using one-way ANOVA with a significance level of $p<0.05$. Post hoc comparison among groups was performed using the least significant difference method.

\section{RESULTS}

\section{Experimental Design and Physiological Parameters}

Illustration of the experimental timelines (Figure 1A). There were no significant differences in CBF (Figures 1B,C), blood MAP, $\mathrm{pO}_{2}$, or $\mathrm{pCO}_{2}$ (Table 1) between these groups.

\section{Brain Infarction and Correlates}

A large infarct volume (42.5\%) was seen following $2 \mathrm{~h}$ MCAO and 3 days reperfusion. Both mild $\left({ }^{* *} p<0.01\right)$ and intense $\left({ }^{*} p<0.05\right)$ exercise significantly decreased infarct volumes (32.3\% vs. $26.3 \%$, respectively; Figures 1D,E). Neurological deficits were detected by the 5- (Figure 1F) or 12- (Figure 1G) point score systems; compared to the control group, deficits were decreased significantly $\left({ }^{*} p<0.05\right)$ after either mild or intense exercise. Apoptotic cell death was detected at 3 days as described above; both mild and intense exercise significantly $\left.{ }^{* * *} p<0.001\right)$ decreased cell death $(0.07$ and $0.14 \mathrm{ng} / \mathrm{ml}$, respectively, vs. $0.22 \mathrm{ng} / \mathrm{ml}$ ), but a further significant decrease was noted $\left({ }^{* * *} p<0.001\right)$ in the mild exercise group (Figure $\mathbf{1 H}$ ). Also, a significant $\left({ }^{* * *} p<0.001\right)$ increase in weight was seen in both exercise groups, with mild exercise rats demonstrating additional gain (Figure 1I).

\section{Functional Outcomes}

As shown in Figure 2A, the time taken to fall off the grid was significantly reduced after both mild $(p<0.01)$ or intense $(p<0.05)$ exercise as compared to rest at $3,7,14,21$, and 28 days (Figure 2A); this reduction was significantly more pronounced in mild exercise rats on 7,21 , and 28 days. Similar results were observed in beam balance (Figure 2B), Rota-rod (Figure 2C), adhesive removal (Figures 2D-E), and forelimb placing tests (Figure 2F). On assessment using the Morris water maze (Figures 2G-J) at 24-28 days, exercised rats demonstrated significantly shorter latency to locate the hidden platform as compared to rested controls, with mildly exercised rats attaining significantly better outcomes (Figures 2G-H). Exercised rats spent more time $\left({ }^{*} p<0.05\right)$ in the target quadrant to find the hidden submerged platform than rested rats (Figure 2I). In contrast, there was no significant difference
TABLE 1 | Physiological parameters during surgery.

\begin{tabular}{lccc}
\hline & Stroke & Intense exercise & Mild exercise \\
\hline MAP (mm Hg) & & & \\
$\quad$ Prior to MCAO & $86.8 \pm 3.3$ & $87.0 \pm 3.5$ & $87.5 \pm 3.4$ \\
Onset of reperfusion & $86.7 \pm 2.4$ & $86.8 \pm 2.5$ & $86.4 \pm 3.5$ \\
$\quad$ After reperfusion & $82.3 \pm 2.9$ & $85.3 \pm 3.1$ & $85.8 \pm 4.0$ \\
$\mathrm{pCO}_{2}$ (mm Hg) & & & \\
Prior to MCAO & $44.8 \pm 1.7$ & $45.0 \pm 2.2$ & $47.2 \pm 3.5$ \\
Onset of reperfusion & $42.4 \pm 2.7$ & $45.4 \pm 2.0$ & $43.2 \pm 2.1$ \\
After reperfusion & $45.2 \pm 4.0$ & $44.3 \pm 2.7$ & $43.4 \pm 4.4$ \\
$\mathrm{pO}_{2}$ (mm Hg) & & & \\
Before MCAO & $132.9 \pm 5.9$ & $139.5 \pm 5.6$ & $132.4 \pm 5.7$ \\
Onset of reperfusion & $135.2 \pm 5.1$ & $132.7 \pm 5.6$ & $134.7 \pm 4.9$ \\
After reperfusion & $134.1 \pm 9.1$ & $138.2 \pm 6.4$ & $132.4 \pm 4.1$ \\
\hline
\end{tabular}

MAP, mean arterial pressure; MCAO, middle cerebral artery occlusion.

between groups concerning swim speed, suggesting similar gross motor skills (Figure 2J). These results demonstrate the significant role of exercise generally, and mild exercise in particular, in the long-term recovery of sensorimotor functions and spatial learning capability after ischemia/reperfusion injury.

\section{Neuroplasticity}

Flow cytometry assay demonstrated that both mild $\left({ }^{* *} p<0.01\right)$ and intense $\left({ }^{* *} p<0.01\right)$ exercise increased expression of Tau (Figures 3A,C) and GAP-43 (Figures 3B,D) at 3, 14, and 28 days; significantly more Tau expression was seen in mildly exercised rats $\left({ }^{* *} p<0.01\right)$. Also, compared to the control group, mild and intense exercise both significantly increased protein expression of Tau, GAP-43, and PSD-95 at 3, 14, and 28 days. Compared to the control group, levels of Tau ( ${ }^{* *} p<0.01$, Figure 3E), GAP-43 $\left({ }^{* * *} p<0.001\right.$, Figure 3F), PSD-95 ( ${ }^{*} p<0.05$, Figure 3G), and SYN (Figure 3H) were found by Western Blot to be increased in mildly exercised rats at 3,14 , and 28 days; the same results were also seen in the intense exercise group. Taken together, these results demonstrate the capacity of exercise to augment neuroplasticity after ischemia/reperfusion injury.

\section{Expression of HIF-1 $\alpha$, BDNF, TrkB, and CREB}

Both exercise protocols yielded a significant increase in levels of these proteins at 3,14 , and 28 days. Compared to the control group, levels of HIF- $1 \alpha$ ( 3 days, ${ }^{*} p<0.05 ; 28$ days, ${ }^{* *} p<0.01$, Figure 4A), BDNF (Figure 4B), NGF (14 days and 28 days, ${ }^{* * *} p<0.001$, Figure 4C), TrkB $\left({ }^{*} p<0.05\right.$, Figure 4D), and CREB ( ${ }^{* *} p<0.01$, Figure 4E) were significantly increased in both the mild and intense exercise groups. Levels of HIF-1 $\alpha$ ( 3 days, ${ }^{*} p<0.05$, Figure 4A), BDNF (14 days, ${ }^{* *} p<0.01$, Figure 4B), NGF (3 days, ${ }^{* *} p<0.01$, Figure 4C), TrkB (14 days, ${ }^{*} p<0.05$, Figure 4D), and CREB (Figure 4E) were further increased in mildly exercised rats. These results demonstrate the alterations in HIF-1 $\alpha$, BDNF, TrkB, and CREB in association with synaptic plasticity following ischemia/reperfusion injury.

\section{DISCUSSION}

The results obtained in this study, confirmed the augmentation in neuroplasticity in the ipsilesional hemisphere and functional 

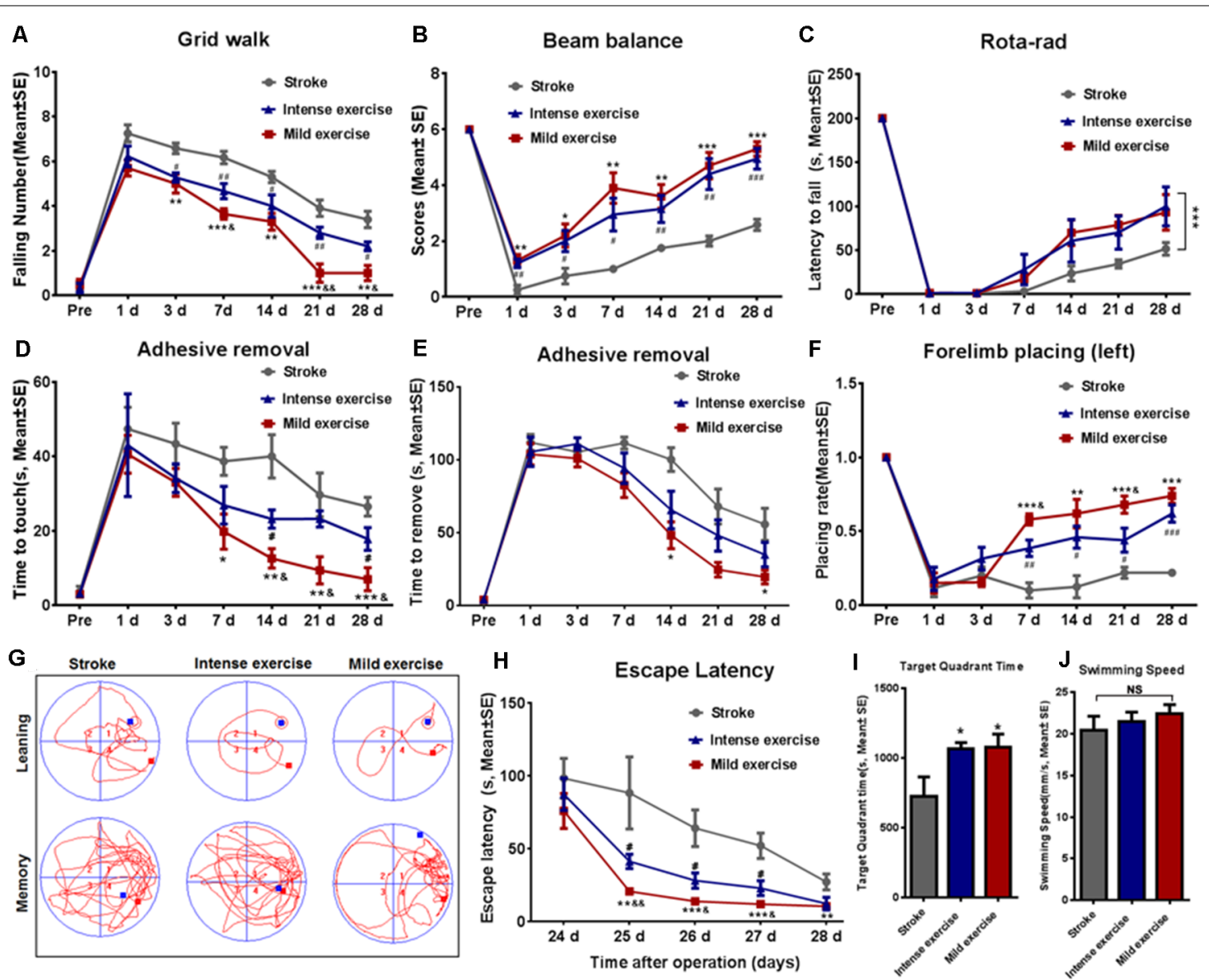

FIGURE 2 | Exercise-mediated enhancement of functional recovery. (A) Grid walk test. Foot slips from the grid were significantly reduced after both mild (5.0 vs. 6.6 at 3 days, ${ }^{* *} p<0.01 ; 3.6$ vs. 6.2 at 7 days, ${ }^{* \star *} p<0.001 ; 3.3$ vs. 5.3 at 14 days, ${ }^{* \star} p<0.01 ; 1.0$ vs. 3.9 at 21 days, ${ }^{* * *} p<0.001 ; 1.0$ vs. 3.4 at 28 days, ${ }^{* *} p<0.01$ ) and intense (5.2 vs. 6.6 at 3 days, ${ }^{\#} p<0.05 ; 4.7$ vs. 6.2 at 7 days, ${ }^{\# \#} p<0.01 ; 4.0$ vs. 5.3 at 14 days, ${ }^{\#} p<0.05 ; 2.8$ vs. 3.9 at 21 days, ${ }^{\#} p<0.05$; 2.2 vs. 3.4 at 28 days, $\# p<0.01$ ) exercise rats as compared to control rats at $3,7,14,21$, and 28 days. Mild exercise conferred further benefit over intense exercise in this respect (5.7 vs. 6.2 at 1 day; 5.0 vs 5.2 at 3 days; 3.6 vs. 4.7 at 7 days, ${ }^{\&} p<0.05 ; 3.3$ vs. 4.0 at 14 days; 1.0 vs. 2.8 at 21 days, ${ }^{\text {\& }} p<0.01 ; 1.0$ vs. 2.2 at 28 days, $\left.{ }^{\&} p<0.05\right)$. Similar results were observed in beam balance (B), Rota-rod (C), adhesive removal (D,E), and forelimb placing tests (F). Learning ability was examined by the Morris water maze test at 24-28 days of exercise (G-J). Representative images of the swim paths at 28 days (G). Latency to locate the submerged platform at $24-28$ days $\mathbf{( H )}$. Target quadrant time $\mathbf{( I )}$ and swim speed $(\mathbf{J})$ at 28 days. ${ }^{*} p \leq 0.05,{ }^{* \star} p \leq 0.01,{ }^{* \star} p \leq 0.001$ represent mild exercise vs. control; $\# p \leq 0.05,{ }^{\# \#} p \leq 0.01,{ }^{\# \#} p \leq 0.001$ represent intense exercise vs. control; ${ }^{\circledR} p \leq 0.05$ represent intense exercise vs. mild exercise. NS, not significant.

outcomes provided by physical exercise after ischemic brain injury. Specifically, we showed that both mild and intense exercise regimens reduced brain infarct volume and apoptotic cell death, and improved motor and cognitive function at 3, 14 , and 28 days after ischemia/reperfusion injury. The early improvement in infarct volume seen in these results aligned with a previous meta-analysis, in which infarct volume was reduced most effectively by exercise administered with the shortest delays after ischemia (Egan et al., 2014); data from our group derived from pre-conditioning experimentation suggest that this may be related to the capacity of exercise to mitigate inflammatory damage during reperfusion (Ding et al., 2005), with the caveat that the exercise initiation too early after ischemia may be detrimental (Li et al., 2017a). More recent work by our group further substantiated these findings by demonstrating that exercise improved glycometabolism in the ischemic area and decreased neuroinflammation and apoptosis as early as 1 day post-stroke, and also at 3 days (Shen et al., 2016; Li et al., $2017 \mathrm{a}, \mathrm{b}, \mathrm{c})$. These findings suggest that it is beneficial to initiate exercise early after ischemia/reperfusion, as was done in the present study.

Furthermore, our biochemical analyses showed that the expression of synaptic plasticity proteins (Tau, GAP-43, and PSD-95) and their potential upstream regulators (HIF-1 $\alpha$, BDNF, NGF, TrkB, and CREB) were significantly increased after exercise. These findings suggest that long-term physical exercise may induce synaptic plasticity through the HIF-1 $\alpha$ and $\mathrm{BDNF} / \mathrm{TrkB} / \mathrm{CREB}$ pathway. Brain synaptic regeneration may be related to elevated levels of GAP-43 or Tau proteins, and exercise has been shown to increase expression of GAP-43 in the ischemic area in rats with cerebral ischemia/reperfusion injury (Mizutani et al., 2014). Exercise-induced GAP-43 has 

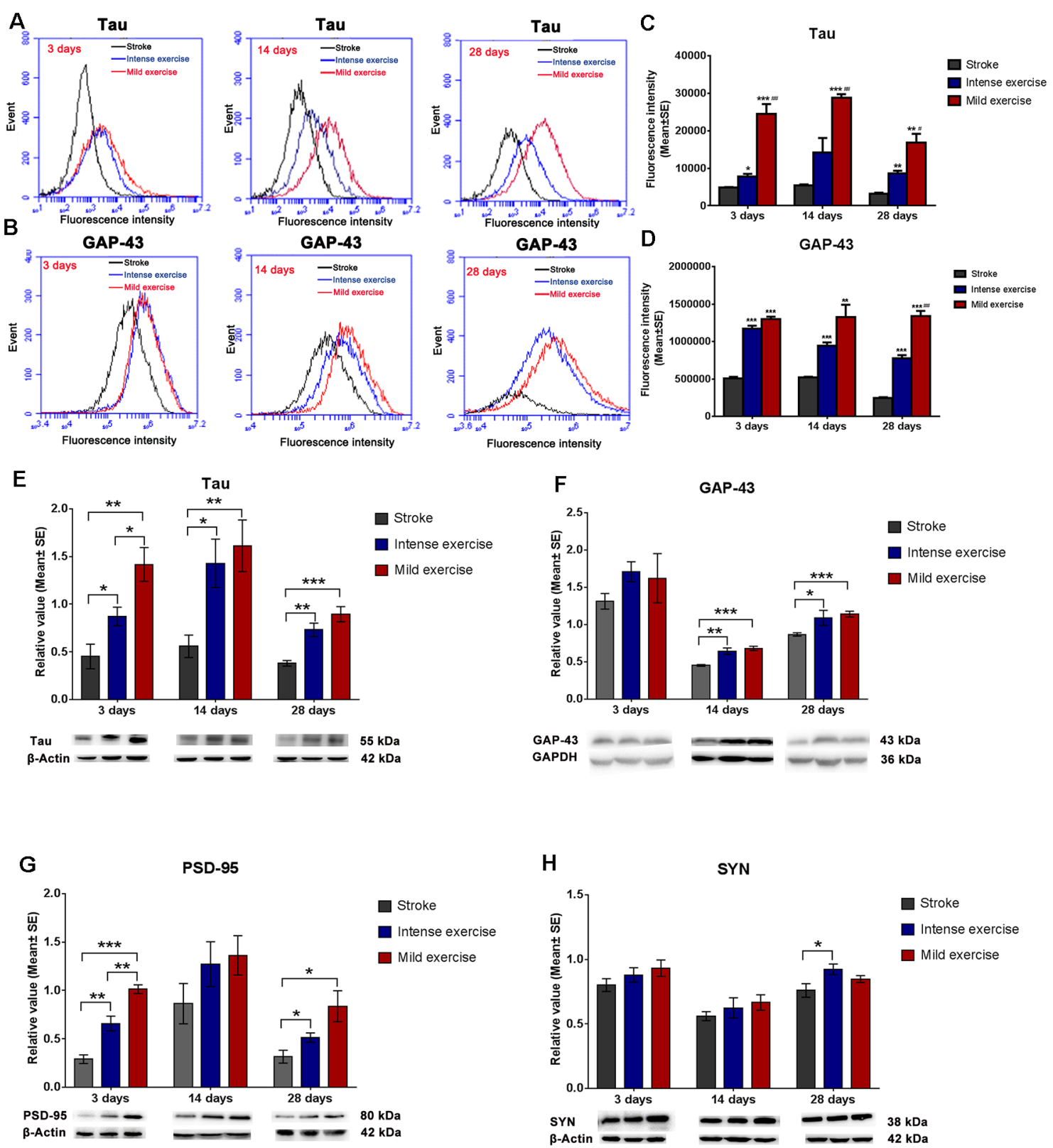

FIGURE 3 | Exercise-induced increased expression of synaptic proteins. (A-D) Representative images of Tau and GAP-43 detected by FCM. Both mild (A,C $24,600.2$ vs. $4,885.12$ at 3 days, ${ }^{* \star *} p<0.001 ; 28,897$ vs. $5,408.9$ at 14 days, ${ }^{* \star *} p<0.001 ; 16,879.2$ vs. $3,186.86$ at 28 days, $\left.{ }^{* *} p<0.01\right)$ and intense $(7,752.19$ vs. $4,885.12$ at 3 days, ${ }^{*} p<0.05 ; 14,230.5$ vs. $5,408.9$ at 14 days; $8,698.12$ vs. $3,186.86$ at 28 days, ${ }^{\star *} p<0.01$ ) exercise significantly induced Tau expression at 3,14 , and 28 days. Further increases in expression were seen in mildly exercised rats at 3,14 , and 28 days $\left(" 0.05,{ }^{\# \#} 0.01, \# \# 0.001\right.$ represent mild exercise vs. intense exercise). The same results were also seen for GAP-43 expression (B,D). (E-H) Representative images of Tau, GAP-43, PSD-95, and SYN as detected by Western Blot. Compared to the control group, levels of Tau (E; 1.4 vs. 0.5 at 3 days, ${ }^{* *} p<0.01 ; 1.6$ vs. 0.6 at 14 days, ${ }^{* *} p<0.01 ; 0.9$ vs. 0.4 at 28 days, $\left.{ }^{* \star *} p<0.001\right)$, GAP-43 (F; 1.6 vs. 1.3 at 3 days; 0.7 vs. 0.5 at 14 days, ${ }^{* *} p<0.001 ; 1.1$ vs. 0.8 at 28 days, $\left.{ }^{* * *} p<0.001\right)$, PSD-95 (G; 1.0 vs. 0.3 at 3 days, ${ }^{* * *} p<0.001 ; 1.4$ vs. 0.9 at 14 days; 0.8 vs. 0.3 at 28 days, ${ }^{*} p<0.05$ ), and SYN ( $\mathbf{H} ; 0.9$ vs. 0.8 at 3 days; 0.7 vs. 0.6 at 14 days; 0.8 vs. 0.8 at 28 days) in mildly exercised rats were increased. The same results were also seen with intense exercise. Levels of Tau (E; 1.4 vs. 0.9 at 3 days, ${ }^{*} p<0.05 ; 1.6$ vs. 1.4 at 14 days; 0.9 vs. 0.7 at 28 days), GAP-43 (F; 1.6 vs. 1.7 at 3 days; 0.7 vs. 0.6 at 14 days; 1.1 vs. 1.0 at 28 days), PSD-95 (G; 1.0 vs. 0.7 at 3 days, ${ }^{\star \star} p<0.01 ; 1.4$ vs. 1.3 at 14 days; 0.8 vs. 0.5 at 28 days), and SYN (H; 0.9 vs. 0.9 at 3 days; 0.7 vs. 0.6 at 14 days; 0.8 vs. 0.9 at 28 days) were similar between mildly and intensely exercised rats.

been associated with augmented hippocampal neuroplasticity (Liu W. et al., 2018; Rahmati and Kazemi, 2019) in a process that appears to be dependent on BDNF maturation and the TrkB signaling promoted by mature BDNF (Ding et al., 2011).
Similarly, exercise has been shown to promote axonal recovery as assessed by the upregulation of Tau and GAP-43 and is associated with functional improvement after cerebral infarction (Li et al., 2015). Short-term moderate exercise also appears to be 


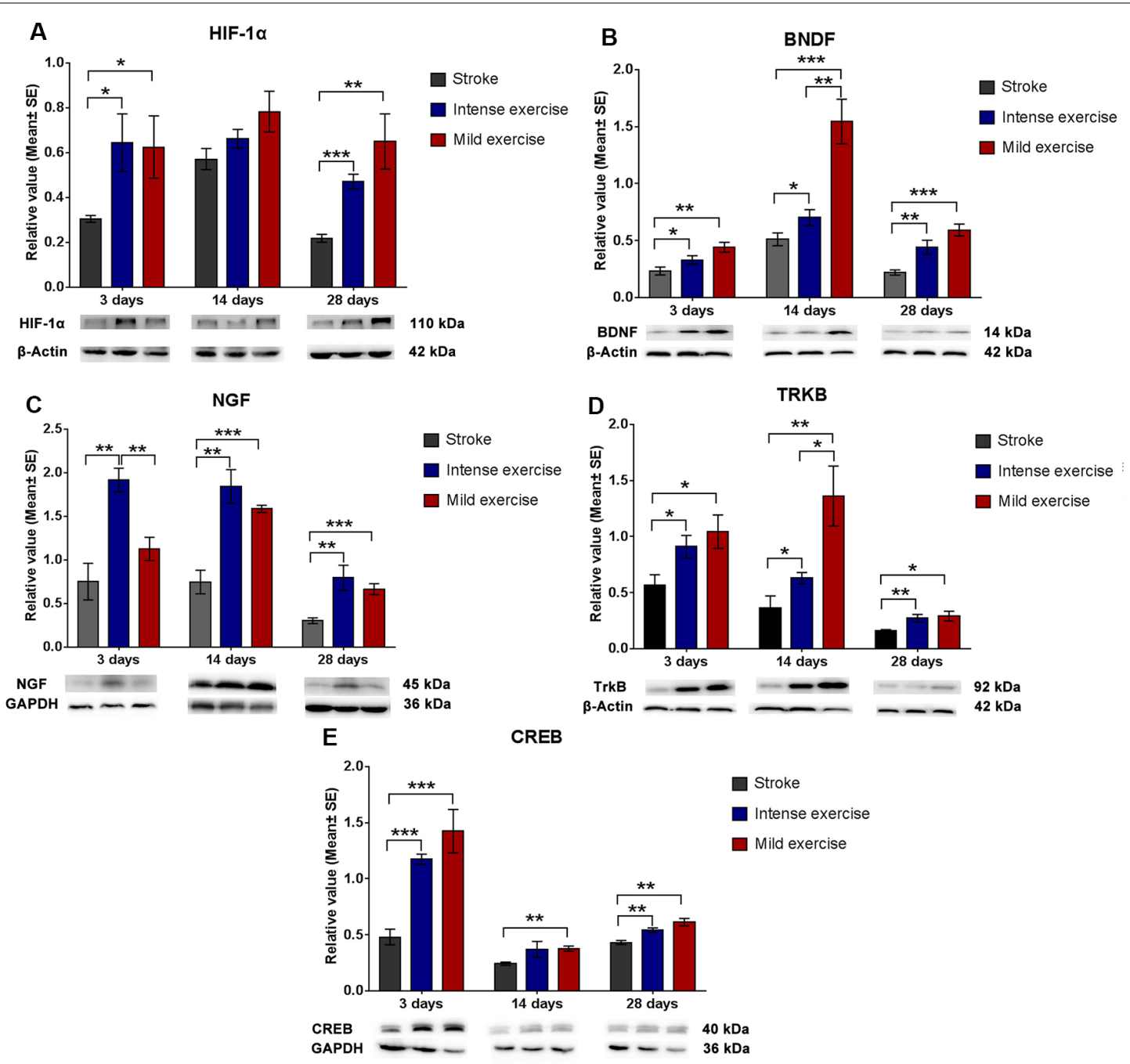

FIGURE 4 | Augmented HIF-1 $\alpha /$ BDNF/ TrkB/CREB pathway protein expression after exercise. Compared to rested rats, levels of HIF- $1 \alpha$ (A; 0.6 vs. 0.3 at 3 days, ${ }^{*} p<0.05 ; 0.8$ vs. 0.6 at 14 days; 0.7 vs. 0.2 at 28 days, ${ }^{* \star} p<0.01$ ), BDNF (B; 0.4 vs. 0.2 at 3 days; 1.5 vs. 0.5 at 14 days; 0.6 vs. 0.2 at 28 days), NGF (C; 1.1 vs. 0.7 at 3 days; 1.6 vs. 0.7 at 14 days, ${ }^{* \star *} p<0.001 ; 0.6$ vs. 0.3 at 28 days, ${ }^{* * *} p<0.001$ ), TrkB (D; 1.0 vs. 0.6 at 3 days, ${ }^{*} p<0.05 ; 1.4$ vs. 0.4 at 14 days, ${ }^{* *} p<0.01 ; 0.3$ vs. 0.2 at 28 days, ${ }^{*} p<0.05$ ), and CREB (E; 1.4 vs. 5.5 at 3 days, ${ }^{* \star *} p<0.001 ; 0.4$ vs. 0.2 at 14 days, ${ }^{* *} p<0.01 ; 0.6$ vs. 0.4 at 28 days, ${ }^{* *} p<0.01$ ) in mildly exercised rats were significantly increased. The same results were seen in intensely exercised rats. Levels of HIF-1 1 (A; 0.6 vs. 0.6 at 3 days, ${ }^{*} p<0.05$; 0.8 vs. 0.7 at 14 days; 0.7 vs. 0.5 at 28 days), BDNF (B; 0.4 vs. 0.3 at 3 days; 1.5 vs. 0.7 at 14 days, ${ }^{* *} p<0.01 ; 0.6$ vs. 0.4 at 28 days), NGF (C; 1.1 vs. 1.9 at 3 days, ${ }^{\star *} p<0.01 ; 1.6$ vs. 1.8 at 14 days; 0.6 vs. 0.8 at 28 days), TrkB (D; 1.0 vs. 0.9 at 3 days; 1.4 vs. 0.6 at 14 days, ${ }^{*} p<0.05 ; 0.3$ vs. 0.3 at 28 days), and CREB (E; 1.4 vs. 1.2 at 3 days; 04 vs. 0.4 at 14 days; 0.6 vs. 0.5 at 28 days) were similar between exercise intensities.

capable of inducing the BDNF-regulated marker of hippocampal and structural plasticity known as SYN (Ferreira et al., 2011). One critical component of synaptic plasticity, the dynamic reorganization of the PSD protein scaffold (Coley and Gao, 2019), is augmented by yet another synaptic protein, PSD95. PSD-95 is constituent of the postsynaptic membrane that plays a key role in the plasticity and structure of the excitatory chemical synapse (Wu et al., 2017), and multiple studies have associated physical exercise with its induction (Jung and Kim, 2017; Pan et al., 2017). A series of studies have shed light on the relationship between these factors by demonstrating that BDNF/NGF participate in promoting neuroplasticity for motor rehabilitation after focal cerebral infarction (Matsuda et al., 2011; Mizutani et al., 2011; Mang et al., 2013); BDNF was reported to be induced by exercise, and may regulate the expression of synaptic proteins including GAP-43 (Liu W. et al., 2018), SYN (Ferreira et al., 2011), PSD-95 (Li X. et al., 2019) and Tau (Kerling et al., 2017). This research indicates that synaptic plasticity after stroke is determined, at least in part, by the induction and upregulation of axonal or synaptic proteins that, in our study, were found to be increased in both exercise cohorts.

Also, our work helps to elucidate the role occupied by another factor: HIF-1 $\alpha$. The present results are consistent with previous studies showed that the upregulation of HIF$1 \alpha$ promoted synapse plasticity by mediating synaptic markers 
(Li G. et al., 2019), and played a beneficial role in post-stroke exercise inducing angiogenesis and neurogenesis (Li C. et al., 2017). Recent work demonstrates that exercise could activate the cerebral motor and cognitive circuits by increasing the expression of HIF-1 $\alpha$, suggesting a regulatory role of HIF$1 \alpha$ in exercise-enhanced neuroplasticity (Halliday et al., 2019). Additionally, previous studies also indicated that HIF- $1 \alpha$ regulated BDNF (Chen et al., 2017), TrkB (Martens et al., 2007), and CREB (Yu et al., 2020). Furthermore, the activation of the $\mathrm{BDNF} / \mathrm{TrkB} / \mathrm{CREB}$ pathway has been reported to contribute to the reduction in cerebral ischemic injury and improvement in functional recovery after stroke (Liu H. et al., 2018). Therefore, the enhanced expression of HIF-1 $\alpha$ and $\mathrm{BDNF} / \mathrm{TrkB} / \mathrm{CREB}$ proteins after ischemia/reperfusion injury in the present study suggest that HIF-1 $\alpha$ might be involved in the BDNF pathway, known to promote synaptic plasticity. Although, we did not explicitly study the regulation of HIF$1 \alpha$ on the BDNF/TrkB/CREB pathway, our results suggest a potential link between the molecules. Our findings could be a basis to further clarify the participation of HIF- $1 \alpha$ in BDNF-mediated synaptogenesis.

The results obtained in this experiment are in agreement with previous studies showing that exercise improves motor and cognitive function (Chen et al., 2019; Palasz et al., 2019; Tíglás et al., 2019). Our results also, by suggesting the adequacy of milder post-stroke exercise, address the controversy in the literature regarding the dependence of these beneficial effects on the intensity of the prescribed exercise regimens (Han et al., 2017). A previous investigation supports the findings in the present study, by reporting that higher intensity exercise can exacerbate brain injury after ischemia, whereas the effects of mild intensity training were found to be encouraging (Scopel et al., 2006). Additional work has shown that cell proliferation in the dentate gyrus (Kim et al., 2003), spatial memory function (Lee et al., 2009), and synaptic plasticity (Shih et al., 2013) were more remarkable with mild rather than with heavy exercise after ischemia. In contrast, high-intensity intermittent exercise (HIT) was reported to be superior to moderate-intensity continuous training (MCT) in improving neural plasticity after cerebral ischemia in rats (Pin-Barre et al., 2017; Luo et al., 2019). HIT had a similar effect on cardiac troponin-I as workloadmatched continuous exercise in endurance runners, which could be considered as high intensity exercise (Li et al., 2020) and was reported to be acceptable in stroke patients (Boyne et al., 2016). In agreement with these studies, our present results support the beneficial effect of intense exercise, but also indicate that mild exercise is not necessarily worse; and may be adequate to augment neuroprotection and neuroplasticity after stroke. Further investigation is needed in order to optimize exercise intensity post-stroke and determine which intensity may be most beneficial for neurorehabilitation. Furthermore, a standardized definition of exercise intensities may be necessary in order to homogenize methodologies and better compare results between studies in the future.

The protocol utilized in the present study to define exercise intensities was based on prior works (Curry et al., 2010; Zhang et al., 2012). We employed as a standard of achieved exercise training intensity the speed at which rats could not run any longer due to fatigue within three minutes after the onset of exercise. The therapeutic doses of physical exercise training used in our study were calculated as $40 \%$ of this maximum velocity in the case of mild exercise training, which amounted to approximately $15 \mathrm{~m} / \mathrm{min}$, and $80 \%$ in the case of intense exercise training, which was about $32 \mathrm{~m} / \mathrm{min}$ (Zhang et al., 2012). To further increase the difference between our categories, we reduced speed in the mild group to a maximum of $12 \mathrm{~m} / \mathrm{min}$ as previous studies (Tian et al., 2013; Zhang P. et al., 2013; Zhang et al., 2017; Tang et al., 2018). For the high-intensity group, we selected $30 \mathrm{~m} / \mathrm{min}$ because we have employed this speed in previous work, in which we found that it reduced brain damage (Ding et al., 2006), blood-brain barrier dysfunction (Guo et al., 2008), and brain inflammation in stroke (Curry et al., 2010). Recently, studies used physiological parameters, transferable to patients, to determine high and low intensity in rats (Pin-Barre et al., 2017; Luo et al., 2019). To agree with the principle that a physical exercise regimen is reproducible (Gronwald et al., 2019), our further exercise procedure would focus on a physiological indicator such as the lactate threshold. Another key finding from our previous work was the influence of initiation time on post-stroke rehabilitation outcomes: initiation $6 \mathrm{~h}$ post-stroke exacerbated brain damage, but this was avoided when exercise was deferred for 1-3 days (Li et al., 2017a,b). Therefore, the initiation time of $24 \mathrm{~h}$ after stroke was selected in this study.

In this study, we intended to observe the protective effects of post-stroke exercise on brain infarct at 3 days as previous studies did. Recent work by our group substantiates these findings by demonstrating exercise-improved glycometabolism, decreased neuroinflammation, and apoptosis (Li et al., 2017a,b,c). The present results were largely supported by the findings of other groups, which demonstrated that exercise accelerated $\mathrm{CBF}$ (Pianta et al., 2019), decreased infarct volume (Tian et al., 2013; Zhang Y. et al., 2013; Pan et al., 2020) and improved functional outcomes (Pianta et al., 2019). In contrast, a few studies have reported no neuroprotective effects of exercise on the neurological deficit and infarct volume after stroke within 3 days (Matsuda et al., 2011; Cui et al., 2020). Future studies are necessary to fully elucidate the effect of post-stroke exercise on brain injury.

Some limitations are important to consider when interpreting the results of our study. The different constant-intensity regimens were used to demonstrate the concept of mild or intense exercise. Although the exercise protocol in rats cannot be directly transferred to patients, in the present study, as the first step, we intended to use these two exercise procedures to investigate the mechanism underlying the dose-dependent benefit of exercise on recovery after stroke. More careful design is on the way to develop a translational strategy that better applies to human stroke patients (Gronwald et al., 2019). For this purpose, our future work will focus on a connection between animal and clinical exercise procedures by controlling the workload of each training regimen as well as using lactate threshold or oxygen uptake as an indicator. Regarding the interpretation of the neurobehavioral test, possibly the multiple 
Rotarod tests served as a training procedure that may have influenced our results. Given our study's focus on the effect and mechanism of different exercise doses on rehabilitation, in each group, the rats received the same test. It is unlikely that this small amount of possible training would change the direction of our results.

In conclusion, this study demonstrates the positive effect on brain injury, functional outcome, and neuroplasticity conferred by both mild and intense long-term treadmill exercise. Additionally, our results suggest that intense exercise did not confer further benefit when compared with its milder counterpart, thus mild exercise may be adequate and sufficient to elicit rehabilitative benefits post-stroke. Moreover, the results may provide a base for our future study regarding the regulation of HIF- $1 \alpha$ on the BDNF/TrkB/CREB pathway in the biochemical processes underlying post-stroke synaptic plasticity.

\section{DATA AVAILABILITY STATEMENT}

All datasets generated for this study are included in the article/Supplementary Material.

\section{ETHICS STATEMENT}

The animal study was reviewed and approved by the Animal Care and Use Committee of the Capital Medical University.

\section{REFERENCES}

Andrews, S. C., Curtin, D., Hawi, Z., Wongtrakun, J., Stout, J. C., and Coxon, J. P. (2020). Intensity matters: high-intensity interval exercise enhances motor cortex plasticity more than moderate exercise. Cereb. Cortex 30, 101-112. doi: $10.1093 /$ cercor/bhz075

Belayev, L., Alonso, O. F., Busto, R., Zhao, W., and Ginsberg, M. D. (1996). Middle cerebral artery occlusion in the rat by intraluminal suture. Neurol. patholog. eval. improved model Stroke 27, 1616-1623; discussion 1623. doi: 10.1161/01.str.27.9.1616.

Bell, J. A., Wolke, M. L., Ortez, R. C., Jones, T. A., and Kerr, A. L. (2015). Training intensity affects motor rehabilitation efficacy following unilateral ischemic insult of the sensorimotor cortex in C57BL/6 mice. Neurorehabil. Neural Repair 29, 590-598. doi: 10.1177/1545968314553031

Belviranli, M., and Okudan, N. (2019). Voluntary, involuntary and forced exercises almost equally reverse behavioral impairment by regulating hippocampal neurotrophic factors and oxidative stress in experimental Alzheimer's disease model. Behav. Brain Res. 364, 245-255. doi: 10.1016/j.bbr.2019. 02.030

Biundo, F., Del Prete, D., Zhang, H., Arancio, O., and D'Adamio, L. (2018). A role for tau in learning, memory and synaptic plasticity. Sci. Rep. 8:3184. doi: 10.1038/s41598-018-21596-3

Boyne, P., Dunning, K., Carl, D., Gerson, M., Khoury, J., Rockwell, B., et al. (2016). High-intensity interval training and moderate-intensity continuous training in ambulatory chronic stroke: feasibility study. Phys. Ther. 96, 1533-1544. doi: $10.2522 /$ ptj.20150277

Chen, F. T., Chen, Y. P., Schneider, S., Kao, S. C., Huang, C. M., and Chang, Y. K. (2019). Effects of exercise modes on neural processing of working memory in late middle-aged adults: an fMRI study. Front. Aging Neurosci. 11:224. doi: 10.3389/fnagi.2019.00224

Chen, C., Jiang, W., Liu, Z., Li, F., Yang, J., Zhao, Y., et al. (2018). Splenic responses play an important role in remote ischemic preconditioning-

\section{AUTHOR CONTRIBUTIONS}

FL conducted the animal and biochemical experiments employed in this research. FL, XG, CH, CS, and YD were instrumental in preparing or revising the manuscript. YD was responsible for the experimental design, in addition to assisting with manuscript preparation and revision.

\section{FUNDING}

This work was partially supported by the National Natural Science Foundation of China (FL, 81802231 and XG, 81871838), the Organization Department of Beijing talents project (FL, 2018000082595G485), the Beijing Tongzhou District Financial Fund, the Science and Technology Plan of Beijing Tongzhou District (FL, KJ2020CX002 and KJ2019CX004-07).

\section{ACKNOWLEDGMENTS}

We thank Sainan Wang, Menglei Liu, Yanlong Zhao, and Chencheng Zhang for technical assistance.

\section{SUPPLEMENTARY MATERIAL}

The Supplementary Material for this article can be found online at: https://www.frontiersin.org/articles/10.3389/fncel. 2020.00186/full\#supplementary-material.

mediated neuroprotection against stroke. J. Neuroinflammation 15:167. doi: 10.1186/s12974-018-1190-9

Chen, J., Yang, Y., Shen, L., Ding, W., Chen, X., Wu, E., et al. (2017). Hypoxic preconditioning augments the therapeutic efficacy of bone marrow stromal cells in a rat ischemic stroke model. Cell. Mol. Neurobiol. 37, 1115-1129. doi: 10.1007/s10571-016-0445-1

Coley, A. A., and Gao, W. J. (2019). PSD-95 deficiency disrupts PFC-associated function and behavior during neurodevelopment. Sci. Rep. 9:9486. doi: 10.1038/s41598-019-45971-w

Cui, J., Kim, C. S., Kim, Y., Sohn, M. K., and Jee, S. (2020). Effects of repetitive transcranial magnetic stimulation (rTMS) combined with aerobic exercise on the recovery of motor function in ischemic stroke rat model. Brain Sci. 10:186. doi: 10.3390/brainsci10030186

Curry, A., Guo, M., Patel, R., Liebelt, B., Sprague, S., Lai, Q., et al. (2010). Exercise pre-conditioning reduces brain inflammation in stroke via tumor necrosis factor- $\alpha$, extracellular signal-regulated kinase $1 / 2$ and matrix metalloproteinase-9 activity. Neurol. Res. 32, 756-762. doi: 10.1179/174313209x459101

Ding, Y. H., Mrizek, M., Lai, Q., Wu, Y., Reyes, R. Jr., Li, J., et al. (2006). Exercise preconditioning reduces brain damage and inhibits TNF- $\alpha$ receptor expression after hypoxia/reoxygenation: an in vivo and in vitro study. Curr. Neurovasc. Res. 3, 263-271. doi: 10.2174/156720206778792911

Ding, Y. H., Young, C. N., Luan, X., Li, J., Rafols, J. A., Clark, J. C., et al. (2005). Exercise preconditioning ameliorates inflammatory injury in ischemic rats during reperfusion. Acta Neuropathol. 109, 237-246. doi: 10.1007/s00401-0040943-y

Ding, Q., Ying, Z., and Gómez-Pinilla, F. (2011). Exercise influences hippocampal plasticity by modulating brain-derived neurotrophic factor processing. Neuroscience 192, 773-780. doi: 10.1016/j.neuroscience.2011.06.032

Egan, K. J., Janssen, H., Sena, E. S., Longley, L., Speare, S., Howells, D. W., et al. (2014). Exercise reduces infarct volume and facilitates neurobehavioral recovery: results from a systematic review and meta-analysis of exercise 
in experimental models of focal ischemia. Neurorehabil. Neural Repair 28, 800-812. doi: 10.1177/1545968314521694

Ferreira, A. F., Real, C. C., Rodrigues, A. C., Alves, A. S., and Britto, L. R. (2011). Short-term, moderate exercise is capable of inducing structural, BDNF-independent hippocampal plasticity. Brain Res. 1425, 111-122. doi: 10.1016/j.brainres.2011.10.004

Gronwald, T., de Bem Alves, A. C., Murillo-Rodriguez, E., Latini, A., Schuette, J., and Budde, H. (2019). Standardization of exercise intensity and consideration of a dose-response is essential. Commentary on "Exercise-linked FNDC5/irisin rescues synaptic plasticity and memory defects in Alzheimer's models", by Lourenco et al., published 2019 in Nature Medicine. J. Sport Health Sci. 8, 353-354. doi: 10.1016/j.jshs.2019.03.006

Guo, M., Lin, V., Davis, W., Huang, T., Carranza, A., Sprague, S., et al. (2008). Preischemic induction of TNF- $\alpha$ by physical exercise reduces blood-brain barrier dysfunction in stroke. J. Cereb. Blood Flow Metab. 28, 1422-1430. doi: $10.1038 /$ jcbfm.2008.29

Halliday, M. R., Abeydeera, D., Lundquist, A. J., Petzinger, G. M., and Jakowec, M. W. (2019). Intensive treadmill exercise increases expression of hypoxia-inducible factor $1 \alpha$ and its downstream transcript targets: a potential role in neuroplasticity. Neuroreport 30, 619-627. doi: 10.1097/wnr. 0000000000001239

Han, P., Zhang, W., Kang, L., Ma, Y., Fu, L., Jia, L., et al. (2017). Clinical evidence of exercise benefits for stroke. Adv. Exp. Med. Biol. 1000, 131-151. doi: 10.1007/978-981-10-4304-8_9

Helan, M., Aravamudan, B., Hartman, W. R., Thompson, M. A., Johnson, B. D., Pabelick, C. M., et al. (2014). BDNF secretion by human pulmonary artery endothelial cells in response to hypoxia. J. Mol. Cell. Cardiol. 68, 89-97. doi: 10.1016/j.yjmcc.2014.01.006

Hu, B., Liu, C., Bramlett, H., Sick, T. J., Alonso, O. F., Chen, S., et al. (2004). Changes in trkB-ERK1/2-CREB/Elk-1 pathways in hippocampal mossy fiber organization after traumatic brain injury. J. Cereb. Blood Flow Metab. 24, 934-943. doi: 10.1097/01.wcb.0000125888.56462.a1

Jung, S. Y., and Kim, D. Y. (2017). Treadmill exercise improves motor and memory functions in cerebral palsy rats through activation of PI3K-Akt pathway. J. Exerc. Rehabil. 13, 136-142. doi: 10.12965/jer.1734964.482

Kerling, A., Kück, M., Tegtbur, U., Grams, L., Weber-Spickschen, S., Hanke, A., et al. (2017). Exercise increases serum brain-derived neurotrophic factor in patients with major depressive disorder. J. Affect. Disord. 215, 152-155. doi: 10.1016/j.jad.2017.03.034

Kim, Y. P., Kim, H. B., Jang, M. H., Lim, B. V., Kim, Y. J., Kim, H., et al. (2003). Magnitude- and time-dependence of the effect of treadmill exercise on cell proliferation in the dentate gyrus of rats. Int. J. Sports Med. 24, 114-117. doi: 10.1055/s-2003-38202

Kim, D. M., and Leem, Y. H. (2016). Chronic stress-induced memory deficits are reversed by regular exercise via AMPK-mediated BDNF induction. Neuroscience 324, 271-285. doi: 10.1016/j.neuroscience.2016. 03.019

Lee, H. W., Ahmad, M., Weldrick, J. J., Wang, H. W., Burgon, P. G., and Leenen, F. H. H. (2018). Effects of exercise training and TrkB blockade on cardiac function and BDNF-TrkB signaling postmyocardial infarction in rats. Am. J. Physiol. Heart Circ. Physiol. 315, H1821-H1834. doi: 10.1152/ajpheart. 00245.2018

Lee, S. U., Kim, D. Y., Park, S. H., Choi, D. H., Park, H. W., and Han, T. R. (2009). Mild to moderate early exercise promotes recovery from cerebral ischemia in rats. Can. J. Neurol. Sci. 36, 443-449. doi: 10.1017/s0317167100007769

Li, F., Geng, X., Khan, H., Pendy, J. T. Jr., Peng, C., Li, X., et al. (2017a). Exacerbation of brain injury by post-stroke exercise is contingent upon exercise initiation timing. Front. Cell. Neurosci. 11:311. doi: 10.3389/fncel.2017. 00311

Li, F., Pendy, J. T. Jr., Ding, J. N., Peng, C., Li, X., Shen, J., et al. (2017b). Exercise rehabilitation immediately following ischemic stroke exacerbates inflammatory injury. Neurol. Res. 39, 530-537. doi: 10.1080/01616412.2017. 1315882

Li, F., Shi, W., Zhao, E. Y., Geng, X., Li, X., Peng, C., et al. (2017c). Enhanced apoptosis from early physical exercise rehabilitation following ischemic stroke. J. Neurosci. Res. 95, 1017-1024. doi: 10.1002/jnr.23890

Li, F., Geng, X., Yip, J., and Ding, Y. (2019). Therapeutic target and cell-signal communication of chlorpromazine and promethazine in attenuating blood- brain barrier disruption after ischemic stroke. Cell Transplant. 28, 145-156. doi: 10.1177/0963689718819443

Li, F., Nie, J., Zhang, H., Fu, F., Yi, L., Hopkins, W., et al. (2020). Effects of matched intermittent and continuous exercise on changes of cardiac biomarkers in endurance runners. Front. Physiol. 11:30. doi: 10.3389/fphys.2020.00030

Li, C., Wen, H., Wang, Q., Zhang, C., Jiang, L., Dou, Z., et al. (2015). Exercise training inhibits the nogo-A/NgR1/Rho-a signals in the cortical peri-infarct area in hypertensive stroke rats. Am. J. Phys. Med. Rehabil. 94, 1083-1094. doi: $10.1097 / \mathrm{phm} .000000000000339$

Li, X., Wu, Q., Xie, C., Wang, C., Wang, Q., Dong, C., et al. (2019). Blocking of BDNF-TrkB signaling inhibits the promotion effect of neurological function recovery after treadmill training in rats with spinal cord injury. Spinal Cord 57, 65-74. doi: 10.1038/s41393-018-0173-0

Li, C., Zhang, B., Zhu, Y., Li, Y., Liu, P., Gao, B., et al. (2017). Poststroke constraint-induced movement therapy increases functional recovery, angiogenesis and neurogenesis with enhanced expression of HIF-1 $\alpha$ and VEGF. Curr. Neurovasc. Res. 14, 368-377. doi: 10.2174/1567202614666171128120558

Li, G., Zhao, M., Cheng, X., Zhao, T., Feng, Z., Zhao, Y., et al. (2019). FG-4592 improves depressive-like behaviors through HIF-1mediated neurogenesis and synapse plasticity in rats. Neurotherapeutics doi: 10.1007/s13311-019-00807-3 [Epub ahead of print].

Linder, S. M., Rosenfeldt, A. B., Davidson, S., Zimmerman, N., Penko, A., Lee, J., et al. (2019). Forced, not voluntary, aerobic exercise enhances motor recovery in persons with chronic stroke. Neurorehabil. Neural Repair 33, 681-690. doi: $10.1177 / 1545968319862557$

Liu, W., Xue, X., Xia, J., Liu, J., and Qi, Z. (2018). Swimming exercise reverses CUMS-induced changes in depression-like behaviors and hippocampal plasticity-related proteins. J. Affect. Disord. 227, 126-135. doi: 10.1016/j.jad. 2017.10.019

Liu, H., Zhong, L., Zhang, Y., Liu, X., and Li, J. (2018). Rutin attenuates cerebral ischemia-reperfusion injury in ovariectomized rats via estrogenreceptor-mediated BDNF-TrkB and NGF-TrkA signaling. Biochem. Cell Biol. 96, 672-681. doi: 10.1139/bcb-2017-0209

Luo, L., Li, C., Deng, Y., Wang, Y., Meng, P., and Wang, Q. (2019). High-intensity interval training on neuroplasticity, balance between brainderived neurotrophic factor and precursor brain-derived neurotrophic factor in poststroke depression rats. J. Stroke Cerebrovasc. Dis. 28, 672-682. doi: 10.1016/j.jstrokecerebrovasdis.2018.11.009

Mang, C. S., Campbell, K. L., Ross, C. J., and Boyd, L. A. (2013). Promoting neuroplasticity for motor rehabilitation after stroke: considering the effects of aerobic exercise and genetic variation on brain-derived neurotrophic factor. Phys. Ther. 93, 1707-1716. doi: 10.2522/ptj.20130053

Martens, L. K., Kirschner, K. M., Warnecke, C., and Scholz, H. (2007). Hypoxia-inducible factor-1 (HIF-1) is a transcriptional activator of the TrkB neurotrophin receptor gene. J. Biol. Chem. 282, 14379-14388. doi: 10.1074/jbc. M609857200

Matsuda, F., Sakakima, H., and Yoshida, Y. (2011). The effects of early exercise on brain damage and recovery after focal cerebral infarction in rats. Acta Physiol. 201, 275-287. doi: 10.1111/j.1748-1708.2010.02174.x

Mercerón-Martínez, D., Almaguer-Melian, W., Alberti-Amador, E., and Bergado, J. A. (2018). Amygdala stimulation promotes recovery of behavioral performance in a spatial memory task and increases GAP-43 and MAP-2 in the hippocampus and prefrontal cortex of male rats. Brain Res. Bull. 142, 8-17. doi: 10.1016/j.brainresbull.2018.06.008

Mizutani, K., Sonoda, S., Wakita, H., Katoh, Y., and Shimpo, K. (2011). Functional recovery and alterations in the expression and localization of protein Kinase $\mathrm{C}$ following voluntary exercise in rat with cerebral infarction. Neurol. Sci. 35, 53-59. doi: 10.1007/s10072-013-1477-7

Mizutani, K., Sonoda, S., Yamada, K., Beppu, H., and Shimpo, K. (2011). Alteration of protein expression profile following voluntary exercise in the perilesional cortex of rats with focal cerebral infarction. Brain Res. 1416, 61-68. doi: 10.1016/j.brainres.2011.08.012

Nakamura, K., Tan, F., Li, Z., and Thiele, C. J. (2011). NGF activation of TrkA induces vascular endothelial growth factor expression via induction of hypoxiainducible factor-1 $\alpha$. Mol. Cell. Neurosci. 46, 498-506. doi: 10.1016/j.mcn.2010. 12.002

Palasz, E., Niewiadomski, W., Gasiorowska, A., Wysocka, A., Stepniewska, A., and Niewiadomska, G. (2019). Exercise-induced neuroprotection and recovery of 
motor function in animal models of Parkinson's disease. Front. Neurol. 10:1143. doi: 10.3389/fneur.2019.01143

Pan, X., Jiang, T., Zhang, L., Zheng, H., Luo, J., and Hu, X. (2017). Physical exercise promotes novel object recognition memory in spontaneously hypertensive rats after ischemic stroke by promoting neural plasticity in the entorhinal cortex. Front. Behav. Neurosci. 11:185. doi: 10.3389/fnbeh.2017.00185

Pan, G., Jin, L., Shen, W., Zhang, J., Pan, J., Cheng, J., et al. (2020). Treadmill exercise improves neurological function by inhibiting autophagy and the binding of HMGB1 to Beclin1 in MCAO juvenile rats. Life Sci. 243:117279. doi: 10.1016/j.lfs.2020.117279

Pianta, S., Lee, J. Y., Tuazon, J. P., Castelli, V., Mantohac, L. M., Tajiri, N., et al. (2019). A short bout of exercise prior to stroke improves functional outcomes by enhancing angiogenesis. Neuromolecular Med. 21, 517-528. doi: 10.1007/s12017-019-08533-x

Pin-Barre, C., Constans, A., Brisswalter, J., Pellegrino, C., and Laurin, J. (2017). Effects of high- versus moderate-intensity training on neuroplasticity and functional recovery after focal ischemia. Stroke 48, 2855-2864. doi: 10.1161/strokeaha.117.017962

Pu, H., Shi, Y., Zhang, L., Lu, Z., Ye, Q., Leak, R. K., et al. (2019). Proteaseindependent action of tissue plasminogen activator in brain plasticity and neurological recovery after ischemic stroke. Proc. Natl. Acad. Sci. U S A 116, 9115-9124. doi: 10.1073/pnas.1821979116

Rahmati, M., and Kazemi, A. (2019). Various exercise intensities differentially regulate GAP-43 and CAP-1 expression in the rat hippocampus. Gene 692, 185-194. doi: 10.1016/j.gene.2019.01.013

Ran, Y., Liu, Z., Huang, S., Shen, J., Li, F., Zhang, W., et al. (2018). Splenectomy fails to provide long-term protection against ischemic stroke. Aging Dis. 9, 467-479. doi: 10.14336/ad.2018.0130

Saposnik, G., Cohen, L. G., Mamdani, M., Pooyania, S., Ploughman, M., Cheung, D., et al. (2016). Efficacy and safety of non-immersive virtual reality exercising in stroke rehabilitation (EVREST): a randomised, multicentre, single-blind, controlled trial. Lancet Neurol. 15, 1019-1027. doi: 10.1016/s14744422(16)30121-1

Scopel, D., Fochesatto, C., Cimarosti, H., Rabbo, M., Bello-Klein, A., Salbego, C., et al. (2006). Exercise intensity influences cell injury in rat hippocampal slices exposed to oxygen and glucose deprivation. Brain Res. Bull. 71, 155-159. doi: 10.1016/j.brainresbull.2006.08.011

Shen, J., Huber, M., Zhao, E. Y., Peng, C., Li, F., Li, X., et al. (2016). Early rehabilitation aggravates brain damage after stroke via enhanced activation of nicotinamide adenine dinucleotide phosphate oxidase (NOX). Brain Res. 1648, 266-276. doi: 10.1016/j.brainres.2016.08.001

Shi, Q., Zhang, P., Zhang, J., Chen, X., Lu, H., Tian, Y., et al. (2009). Adenovirusmediated brain-derived neurotrophic factor expression regulated by hypoxia response element protects brain from injury of transient middle cerebral artery occlusion in mice. Neurosci. Lett. 465, 220-225. doi: 10.1016/j.neulet.2009. 08.049

Shih, P. C., Yang, Y. R., and Wang, R. Y. (2013). Effects of exercise intensity on spatial memory performance and hippocampal synaptic plasticity in transient brain ischemic rats. PLoS One 8:e78163. doi: 10.1371/journal.pone. 0078163

Tang, Y., Zhang, Y., Zheng, M., Chen, J., Chen, H., and Liu, N. (2018). Effects of treadmill exercise on cerebral angiogenesis and MT1-MMP expression after cerebral ischemia in rats. Brain Behav. 8:e01079. doi: 10.1002/brb3.1079
Tíglás, T., Németh, Z., Koller, A., Van der Zee, E. A., Luiten, P. G. M., and Nyakas, C. (2019). Effects of long-term moderate intensity exercise on cognitive behaviors and cholinergic forebrain in the aging rat. Neuroscience 411, 65-75. doi: 10.1016/j.neuroscience.2019.05.037

Tian, S., Zhang, Y., Tian, S., Yang, X., Yu, K., Zhang, Y., et al. (2013). Early exercise training improves ischemic outcome in rats by cerebral hemodynamics. Brain Res. 1533, 114-121. doi: 10.1016/j.brainres.2013.07.049

Wang, Z., Hou, L., and Wang, D. (2019). Effects of exercise-induced fatigue on the morphology of asymmetric synapse and synaptic protein levels in rat striatum. Neurochem. Int. 129:104476. doi: 10.1016/j.neuint.2019.104476

Wu, Q., Sun, M., Bernard, L. P., and Zhang, H. (2017). Postsynaptic density 95 (PSD-95) serine 561 phosphorylation regulates a conformational switch and bidirectional dendritic spine structural plasticity. J. Biol. Chem. 292, 16150-16160. doi: 10.1074/jbc.M117.782490

Wu, X., Liu, S., Hu, Z., Zhu, G., Zheng, G., and Wang, G. (2018). Enriched housing promotes post-stroke neurogenesis through calpain 1-STAT3/HIF-1 $\alpha /$ VEGF signaling. Brain Res. Bull. 139, 133-143. doi: 10.1016/j.brainresbull.2018. 02.018

Xing, Y., Yang, S. D., Dong, F., Wang, M. M., Feng, Y. S., and Zhang, F. (2018). The beneficial role of early exercise training following stroke and possible mechanisms. Life Sci. 198, 32-37. doi: 10.1016/j.lfs.2018. 02.018

Yu, Z., Liu, Y., Zhu, J., Han, J., Tian, X., Han, W., et al. (2020). Insights from molecular dynamics simulations and steered molecular dynamics simulations to exploit new trends of the interaction between HIF-1 $\alpha$ and p300. J. Biomol. Struct. Dyn. 38, 1-12. doi: 10.1080/07391102.2019.1580616

Zhang, A., Bai, Y., Hu, Y., Zhang, F., Wu, Y., Wang, Y., et al. (2012). The effects of exercise intensity on $\mathrm{p}-\mathrm{NR} 2 \mathrm{~B}$ expression in cerebral ischemic rats. Can. J. Neurol. Sci. 39, 613-618. doi: 10.1017/s0317167100015341

Zhang, P., Yu, H., Zhou, N., Zhang, J., Wu, Y., Zhang, Y., et al. (2013). Early exercise improves cerebral blood flow through increased angiogenesis in experimental stroke rat model. J. Neuroeng. Rehabil. 10:43. doi: 10.1186/17430003-10-43

Zhang, Y., Zhang, P., Shen, X., Tian, S., Wu, Y., Zhu, Y., et al. (2013). Early exercise protects the blood-brain barrier from ischemic brain injury via the regulation of MMP-9 and occludin in rats. Int. J. Mol. Sci. 14, 11096-11112. doi: 10.3390/ijms140611096

Zhang, Q., Zhang, J., Yan, Y., Zhang, P., Zhang, W., and Xia, R. (2017). Proinflammatory cytokines correlate with early exercise attenuating anxiety-like behavior after cerebral ischemia. Brain Behav. 7:e00854. doi: $10.1002 / \mathrm{brb} 3.854$

Conflict of Interest: The authors declare that the research was conducted in the absence of any commercial or financial relationships that could be construed as a potential conflict of interest.

Copyright (c) 2020 Li, Geng, Huber, Stone and Ding. This is an open-access article distributed under the terms of the Creative Commons Attribution License (CC BY). The use, distribution or reproduction in other forums is permitted, provided the original author(s) and the copyright owner(s) are credited and that the original publication in this journal is cited, in accordance with accepted academic practice. No use, distribution or reproduction is permitted which does not comply with these terms. 